\title{
Soft-tissue reconstruction after total en bloc sacrectomy
}

\author{
Jennifer E. Kim, BS, 1,2 John Pang, BS, Joani M. Christensen, BS, ${ }^{1}$ Devin Coon, MD, ${ }^{1}$ \\ Patricia L. Zadnik, MD, ${ }^{2}$ Jean-Paul Wolinsky, MD, ${ }^{2}$ Ziya L. Gokaslan, MD, ${ }^{2}$ Ali Bydon, MD, ${ }^{2}$ \\ Daniel M. Sciubba, MD, ${ }^{2}$ Timothy Witham, MD, ${ }^{2}$ Richard J. Redett, MD, ${ }^{1}$ and Justin M. Sacks, MD1
}

Departments of ${ }^{1}$ Plastic and Reconstructive Surgery and ${ }^{2}$ Neurosurgery, Johns Hopkins University School of Medicine, The Johns Hopkins Hospital, Baltimore, Maryland

OBJECT Total en bloc sacrectomy is a dramatic procedure that results in extensive sacral defects. The authors present a series of patients who underwent flap reconstruction after total sacrectomy, report clinical outcomes, and provide a treatment algorithm to guide surgical care of this unique patient population.

METHODS After institutional review board approval, data were collected for all patients who underwent total sacrectomy between 2002 and 2012 at The Johns Hopkins Hospital. Variables included demographic data, medical history, tumor characteristics, surgical details, postoperative complications, and clinical outcomes. All subtotal sacrectomies were excluded.

RESULTS Between 2002 and 2012, 9 patients underwent total sacrectomy with flap reconstruction. Diagnoses included chordoma $(n=5)$, osteoblastoma $(n=1)$, sarcoma $(n=2)$, and metastatic colon cancer $(n=1)$. Six patients received gluteus maximus (GM) flaps with a prosthetic rectal sling following a single-stage, posterior sacrectomy. Four required additional paraspinous muscle (PSM) or pedicled latissimus dorsi (LD) fasciocutaneous flaps. Three patients underwent multistage sacrectomy with an anterior-posterior approach, 2 of whom received pedicled vertical rectus abdominis myocutaneous (VRAM) flaps, and 1 of whom received local GM, LD, and PSM flaps. Flap complications included dehiscence $(n=4)$ and infection $(n=1)$. During the 1 st year of follow-up, 2 of 9 patients $(22 \%)$ were able to ambulate with an assistive device by the 1st postoperative month, and 6 of $9(67 \%)$ were ambulatory with a walker by the 3rd postoperative month. By postoperative Month 12, 5 of 9 patients (56\%) - or 5 of 5 patients not lost to follow-up (100\%) -were able to able to ambulate independently.

CONCLUSIONS The authors' experience suggests that the GM and pedicled VRAM flaps are reliable options for softtissue reconstruction of total sacrectomy defects. For posterior-only operations, GM flaps with or without a prosthetic rectal sling are generally used. For multistage operations including a laparotomy, the authors consider the pedicled VRAM flap to be the gold standard for simultaneous reconstruction of the pelvic diaphragm and obliteration of dead space.

http://thejns.org/doi/abs/10.3171/2014.10.SPINE14114

KEY WORDS sacrectomy; soft tissue defect; VRAM; spine surgery; sacral tumor; plastic surgery

$\mathrm{T}$ OTAL en bloc sacrectomy is a dramatic surgical procedure that requires complex bony and soft-tissue reconstruction. Radical oncological resection is warranted for very large, aggressive, benign tumors (i.e., giant cell tumors, osteoblastomas) or low-grade malignant tumors (i.e., chordomas, lymphomas) that extend up to or beyond the first sacral vertebra. Unlike partial or hemisa- crectomy, total sacral amputation results in the bilateral disarticulation of the sacroiliac joint; biomechanical stability is subsequently restored with pelvic reconstruction in which pedicle screw-rod constructs, bony allografts, or vascularized autografts are used. Adequate local or distant soft-tissue flaps are critical for providing a well-vascularized environment to facilitate wound healing. Specific ex-

ABBREVIATIONS AP = anterior-posterior; BMI = body mass index; DM = diabetes mellitus; EBL = estimated blood loss; GM = gluteus maximus; LD = latissimus dorsi; $\mathrm{LOS}=$ length of hospital stay; PSM = paraspinous muscle; $\mathrm{RT}=$ radiation therapy; $\mathrm{VAC}=$ vacuum-assisted closure; VRAM = vertical rectus abdominis myocutaneous. SUBMITTED February 2, 2014. ACCEPTED October 23, 2014.

INCLUDE WHEN CITING Published online March 27, 2015; DOI: 10.3171/2014.10.SPINE14114.

DISCLOSURE Dr. Gokaslan holds an instructorship with AO North America; receives research support from NREF and DePuy; is a stock shareholder with US Spine and Spinal Kinetics; and receives honoraria from the AO Foundation. He holds or has held editorial board memberships at Journal of Neurosurgery: Spine, Spine Journal, Journal of Spinal Disorders, European Spine Journal, Nature Review, World Neurosurgery, and Journal of Surgical Oncology. Dr. Bydon receives research support from DePuy, and he is an Advisory Board Member at Medlmmune LLC. Dr. Sciubba receives research support from DePuy; he also is a consultant at Medtronic, NuVasive, Globus, and DePuy. Dr. Witham receives research support from Eli Lilly \& Co. Dr. Sacks is a speaker/consultant for LifeCell Corp. 
amples of (local) advancement flaps include gluteus maximus (GM) muscle flaps, paraspinous muscle (PSM) flaps, and the pedicled vertical rectus abdominis myocutaneous (VRAM) flaps. The use of the pedicled VRAM simultaneously obliterates dead space and prevents the herniation of small and large bowel from the peritoneal cavity into the posterior operative defect where spinal hardware is routinely placed. Acellular dermal matrix can be used to reconstruct the pelvic floor and prevent posterior herniation of the rectum into the operative defect. ${ }^{21}$

Surgical protocols for reconstruction following total sacral amputation are not routinely standardized. Varying strategies have been described and compared in the literature, including the use of single- or multistage procedures; posterior or anterior-posterior (AP) approaches; avascular or vascularized bone grafts; and local, pedicled, or free flaps. The purpose of this study is to describe a consecutive series of patients who underwent soft-tissue reconstruction after total en bloc sacrectomy, and to report clinical outcomes at 1,3,6, and 12 months postoperatively. We also discuss a variety of surgical strategies for managing total en bloc sacrectomy defects and provide a treatment algorithm that can guide surgical care of this unique patient population.

\section{Methods}

After institutional review board approval, the outcomes of 9 consecutive patients who underwent total en bloc sacrectomy between 2002 and 2012 at The Johns Hopkins Hospital were reviewed. Two of these 9 patients were previously described by Clarke et al., in a study of the posterior-only approach for en bloc sacrectomy. ${ }^{6}$ Any patient who underwent subtotal, partial, or hemisacrectomy-procedures that preserve spinopelvic continuity-was excluded from this study. Data were collected from a retrospective neurosurgical spine tumor database and hospital medical records. The following variables were recorded: patient demographic data, medical history, tumor characteristics, resection and reconstruction details, periprocedural complications, and technical and clinical outcomes.

Preoperative risk factors for wound complications were recorded from patients' electronic medical records and were defined as body mass index (BMI) $>30$, history of diabetes mellitus (DM), smoking, hyperlipidemia, hypercholesterolemia, decreased serum albumin, history of neoadjuvant chemotherapy or radiation therapy (RT), previous spine or abdominal surgery, and prior or concurrent colostomy.

Inclusion criteria for surgery included consenting patients who were medically cleared for surgical intervention, patients with a life expectancy $>3$ months, patients with lesions refractory to chemotherapy and/or RT alone, and patients with neurological impairment or pain from the tumor. Exclusion criteria for surgery included patients with radiosensitive lesions, patients with $<3$ months' life expectancy, and patients who did not meet medical clearance criteria for surgery.

Surgery was performed by a multidisciplinary team of surgical oncologists, neurosurgeons, and plastic and reconstructive surgeons. Total sacrectomy was defined by os- teotomy at the S-1 spinal level or above, with removal of more than one-half of the sacroiliac joint. Lumbar osteotomy was performed if the tumor invaded the lumbar vertebral bodies. Surgical approach varied. The posterior-only approach to the sacrum has been well described. ${ }^{6}$ Briefly, the posterior-only technique involved bilateral iliac osteotomies followed by midline osteotomy or discectomy for en bloc resection of the sacrum. A combined anterior and posterior approach required a staged procedure with dissection of the tumor from the anterior visceral structures, followed by bilateral anterior sacroiliac osteotomies with discectomy. This was followed by the posterior resection and reconstruction. During the anterior stage of the surgical procedure, mobilization of the rectum and colectomy were performed for patients with significant bowel involvement.

In the retrospective chart review, levels of tumor involvement were determined from preoperative CT scans, and osteotomy levels were determined from the surgeon's operative notes and postoperative radiographs and CT scans. En bloc resection was confirmed using the surgeon's operative notes. Operative data, including estimated blood loss (EBL), size of the surgical defect, hardware for spinal reconstruction, and types of soft-tissue flap reconstruction were recorded from the plastic surgery and neurosurgery operative notes. Length of hospital stay (LOS) was defined as the time interval from the date of initial surgery to the discharge date of the same hospitalization.

Postoperative course was documented from patients' electronic medical records in the discharge summary and at standard follow-up visits. Following surgery, patients were seen at 2 weeks and then at 3, 6, 9, and 12 months. Patients were followed up every 6 months in the 2nd year, and then yearly. Clinical outcomes were assessed by ambulation status, wound healing, and complications. Patients were described as having the ability to stand with or without assistance (standing mobility), ambulate with assistance, or ambulate independently. Complications were categorized as general (or nonwound-related) or woundspecific, such as dehiscence of surgical wound, infection, seroma, hematoma, and skin necrosis. Early complications were defined as occurring within 6 weeks of the final stage of the sacrectomy. Delayed complications were defined as occurring after postoperative Week 6.

\section{Results \\ Patient Characteristics}

Between 2002 and 2012, 9 patients underwent total en bloc sacrectomy with flap reconstruction. Table 1 presents the patient demographic data. The mean age of the patients was 52.9 years (range 28-69 years). Four of the patients (44\%) were female and $5(56 \%)$ were male. Preoperative diagnoses included sacral chordoma (5 patients), and osteoblastoma, alveolar soft part sarcoma, pelvic sarcoma, and metastatic colon cancer (1 each). All patients had tumor involvement of S-2 and S-3 vertebrae, with extension up to or beyond S-1. In addition, 2 of the 9 patients had involvement of L-5, and 1 had involvement of both L-4 and L-5.

Perioperative risk factors for wound complications are summarized in Table 2. These included BMI > 30 ( $\mathrm{n}=$ 
TABLE 1. Demographic data and treatment characteristics in 9 patients with en bloc sacrectomy

\begin{tabular}{|c|c|}
\hline Characteristic & Value (\%) \\
\hline Total no. patients & $9(100)$ \\
\hline Male sex & $5(56)$ \\
\hline Mean age in yrs; range & $52.9 ; 28-69$ \\
\hline \multicolumn{2}{|l|}{ Diagnosis } \\
\hline Chordoma & $5(56)$ \\
\hline Pelvic sarcoma & $1(11)$ \\
\hline Alveolar soft part sarcoma & $1(11)$ \\
\hline Osteoblastoma & $1(11)$ \\
\hline Metastatic colon adenocarcinoma & $1(11)$ \\
\hline \multicolumn{2}{|l|}{ Tumor extension } \\
\hline S-1 \& down & $6(67)$ \\
\hline L-5 \& down & $2(22)$ \\
\hline L-4 \& down & $1(11)$ \\
\hline \multicolumn{2}{|l|}{ Approach } \\
\hline Pst & $6(67)$ \\
\hline AP & $3(33)$ \\
\hline \multicolumn{2}{|l|}{ Levels resected } \\
\hline S-1 \& down & $7(78)$ \\
\hline L-5 \& down & $2(22)$ \\
\hline \multicolumn{2}{|l|}{ Fixation } \\
\hline Ant & $0(0)$ \\
\hline Pst & $9(100)$ \\
\hline \multicolumn{2}{|l|}{ Spinal reconstruction } \\
\hline Transiliac bar & $7(78)$ \\
\hline Femoral strut allograft & $9(100)$ \\
\hline Titanium cage & $1(11)$ \\
\hline \multicolumn{2}{|l|}{ Soft-tissue reconstruction } \\
\hline Bilat GM & $7(78)$ \\
\hline Bilat PSM & $3(33)$ \\
\hline Bilat LD & $1(11)$ \\
\hline Synthetic rectal sling & $7(78)$ \\
\hline VRAM & $2(22)$ \\
\hline VAC placement & $4(44)$ \\
\hline
\end{tabular}

Ant $=$ anterior $;$ pst $=$ posterior .

8), DM ( $(n=1)$, tobacco use $(n=1)$, hypertension $(n=4)$, and hyperlipidemia $(\mathrm{n}=3)$. Low serum albumin $(<3.5 \mathrm{~g} /$ dl) was observed in 6 patients. Two patients received preoperative chemotherapy and RT, and 1 received RT only prior to sacrectomy. Two had previously undergone colostomies, whereas a colostomy was created at the time of surgery for 1 patient. Four patients had undergone previous abdominal surgery and 2 had previous spinal surgery; 1 of these patients had received both abdominal and spinal surgery prior to sacrectomy.

\section{Treatment Characteristics}

All 9 patients underwent total en bloc sacral amputation with posterior lumbopelvic fixation and soft-tissue flap reconstruction. Spinal tumor resection and spinopel-
TABLE 2. Perioperative risk factors in 9 patients with en bloc sacrectomy

\begin{tabular}{cc}
\hline \multicolumn{1}{c}{ Risk Factor } & No. (\%) \\
\hline DM & $1(11)$ \\
\hline Smoking & $1(11)$ \\
\hline BMI >30 & $8(89)$ \\
\hline Hypertension & $4(44)$ \\
\hline Hyperlipidemia & $3(33)$ \\
\hline Serum albumin $<3.5 \mathrm{~g} / \mathrm{dl}$ & $6(67)$ \\
\hline Chemotherapy & $2(22)$ \\
\hline Preop & $0(0)$ \\
\hline Postop & $3(33)$ \\
\hline RT & $0(0)$ \\
\hline Preop & $2(22)$ \\
\hline Postop & $4(44)$ \\
\hline Previous/additional op & \\
\hline Spine & $2(22)$ \\
\hline Abdomen & $1(11)$ \\
\hline Colostomy &
\end{tabular}

vic reconstruction were completed by the neurosurgical team, with complex wound closure performed in collaboration with the plastic and reconstructive surgery team. For 2-stage procedures requiring an AP surgical approach, mobilization of the rectum and dissection of the tumor from ventral structures was conducted by general surgery. Treatment characteristics are summarized in Table 1.

\section{Single-Stage Operations}

Six of the 9 operations used a single-stage, posterior approach for resection of spinal levels S-1 and down. For these 6 patients, lumbopelvic reconstruction was accomplished using a femoral strut allograft and transiliac bar (n $=5$ ) or a femoral strut allograft without transiliac fixation $(\mathrm{n}=1)$. For all 6 single-stage operations, complex wound closure was accomplished with bilateral GM flaps and a prosthetic rectal sling composed of acellular dermal matrixes: SurgiMend (TEI Biosciences) $(n=3)$, AlloDerm (LifeCell Corp.) $(\mathrm{n}=2)$, or Veritas (Synovis) $(\mathrm{n}=1)$. Four patients required additional local flaps to reconstruct the extensive sacral defect; this required the mobilization and inset of bilateral PSM flaps in 3 patients, and bilateral pedicled latissimus dorsi (LD) fasciocutaneous flaps in the fourth patient. The mean EBL for single-stage procedures was $4689 \mathrm{ml}$ (range 1500-9000 ml).

\section{Multistage Operations}

Three of 9 patients underwent a multistage sacrectomy performed via an AP approach. Two were completed in 2 stages, whereas 1 required a third stage due to intraoperative hemodynamic instability. Two of these patients required spinal resection from L-5 down, and 1 from S-1 down. Lumbopelvic reconstruction was accomplished with 
a combination of titanium cage, intravertebral rod, transiliac bar, and femoral strut allograft $(n=1)$; transiliac bar and femoral strut allograft $(\mathrm{n}=1)$; or femoral strut allograft alone $(n=1)$. Pedicled VRAM flaps were mobilized in the first stage of all 3 operations and placed into the base of the pelvis; however only 2 of the 3 VRAM flaps were successfully inset during the final stage of the operation. One of the patients developed a pelvic hematoma after the anterior procedure, which led to compression and severe venous congestion of the mobilized VRAM tissue. The flap was discarded, and the wound was subsequently closed with bilateral PSM advancements, pedicled LD flaps based on posterior intercostal perforators, and bilateral GM flaps, along with a human acellular dermal matrix (AlloDerm) rectal sling to prevent sacral herniation. The mean EBL for multistage procedures was $7500 \mathrm{ml}$ (range 5000-9000 $\mathrm{ml})$. Superficial fascia and skin were closed over closedsuction 19-Fr drains placed below the spinal hardware. Patients were placed on postoperative antibiotic prophylaxis and drains were maintained until total output was $<30$ $\mathrm{ml} / 24$ hours while ambulating.

\section{Clinical Outcomes}

Table 3 summarizes postoperative and follow-up outcomes. The mean LOS was 30 days (range 8-64 days); the mean length of postoperative follow-up was 14.3 months (range 1-46 months). Four patients were lost to follow-up before the end of the 1st postoperative year. Three of these patients had transferred to other hospitals and 1 died at 5 months postoperatively. At present, 3 of 9 patients (33\%) are reported to have died of complications related to their disease. The remaining 6 patients were alive at last followup, with no unresolved wound complications.

\section{Functional Outcomes}

Table 4 summarizes patients' ambulatory status at time of follow-up. At 1 month postoperatively, 2 of 9 patients (22\%) were able to ambulate with a rolling walker, 2 of 9 (22\%) were able to stand with assistance, and 5 of $9(56 \%)$ were nonambulatory and confined to a hospital bed. At 3 months postoperatively, 6 of 9 patients $(67 \%)$ seen at follow-up were able to ambulate with a walker, and 1 of $9(11 \%)$ was nonambulatory. By 6 months postoperatively, 4 of 9 patients $(44 \%)$ were able to ambulate independently, and 1 of $9(11 \%)$ was ambulating with a walker. At 12 months postoperatively, 5 of 9 patients $(56 \%)$ - or 5 of 5 patients not lost to follow-up (100\%) -were able to able to ambulate independently.

\section{Wound Complications}

Five of 9 patients (56\%) experienced a wound-related complication during their immediate or long-term postoperative course. Within the first 6 weeks after surgery, 4 of 9 patients (44\%) developed early wound complications. These included wound dehiscence $(n=3)$ and early infection $(n=1)$. One patient with early dehiscence also experienced a deep wound infection. After 6 weeks postoperatively, 1 of 9 patients (11\%) developed delayed dehiscence.

Risk factors associated with the development of postoperative wound complications included the use of a vac- uum-assisted closure (VAC) device, exposure to RT, chemotherapy, and colostomy. ${ }^{4,31}$ A VAC device was used for 4 patients following soft-tissue flap closure. Three of these patients developed wound dehiscence or infection that required at least 1 return to the operating room. A third of patients with prior RT, and half of the patients with prior chemotherapy developed wound dehiscence. One of 3 patients who received a colostomy for bowel diversion prior to or at the time of the procedure developed both wound dehiscence and deep wound infection.

All 4 patients who experienced dehiscence were recipients of bilateral GM flaps. Three of the patients with early dehiscence and the 1 patient with local infection required a return to the operating room for surgical debridement. Neither patient receiving a VRAM flap experienced postoperative wound complications. There were no instances of flap necrosis, local hematoma, or seroma. All patients had a well-healed, sterile wound at last follow-up. In the patient with an abandoned VRAM reconstruction secondary to flap compression, no complications at the harvest site were observed.

\section{Other Complications}

Eight of 9 patients (89\%) experienced nonwound-related complications during the first 6 postoperative weeks. Four patients developed distant infection, all of whom were successfully treated with antibiotics. Infections included urinary tract infection $(\mathrm{n}=3)$ and sepsis $(\mathrm{n}=1)$. Additionally, 1 patient developed systemic inflammatory response syndrome. One patient developed a deep venous thrombosis on postoperative Day 10, which was treated conservatively with anticoagulation. The same patient later experienced femoral allograft failure and required reoperation for debridement and graft removal. One patient developed hyponatremia, and 1 experienced postoperative cardiac arrest necessitating subsequent mechanical ventilation. The patient with metastatic colon adenocarcinoma developed ileus, small bowel obstruction, and a perirectal abscess at the site of rectal resection.

\section{Discussion}

There is currently a lack of evidence guiding management of complex sacral wounds after total en bloc sacrectomy. Oftentimes, methods of soft-tissue reconstruction are based on surgeon preference. We present a series of 9 consecutive patients who underwent flap reconstruction after total en bloc sacrectomy. Despite the dramatic nature of this procedure, postoperative follow-up demonstrated that the majority of patients $(86 \%)$ were able to ambulate with or without assistance by the 3rd postoperative month. By 6 months postoperatively, 100\% (5 of 5) of the patients who were still being followed up were able to ambulate independently or with a rolling walker.

Soft-tissue reconstruction was achieved with either regional flaps (GM, PSM, LD) or with pedicled VRAM flaps. Only patients receiving bilateral local tissue flaps experienced major wound complications necessitating a return to the operating room. Major complications seen in our 2 VRAM flap recipients were related to intraoperative blood loss and hemodynamic instability, as well as bowel com- 


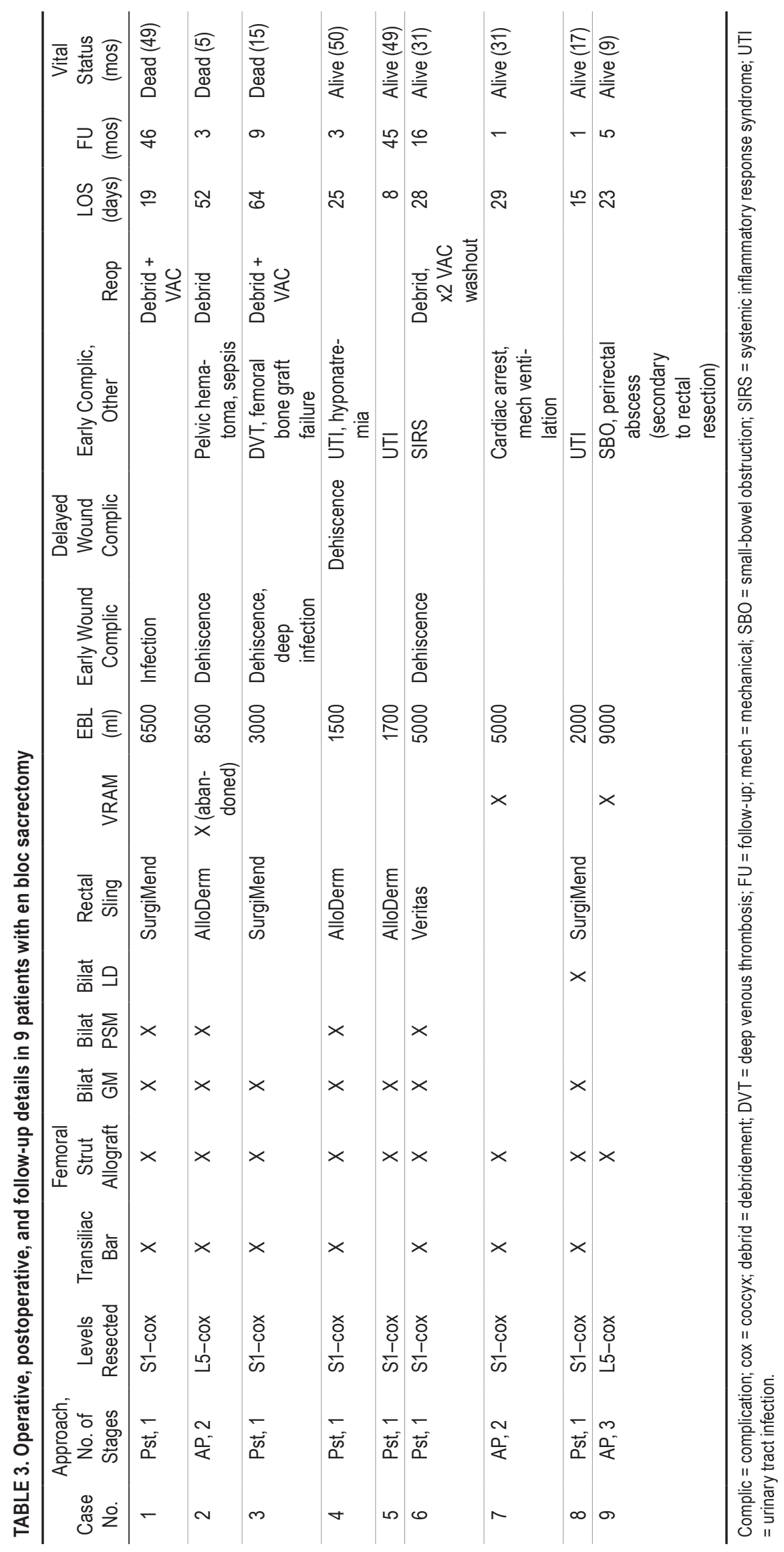


TABLE 4. Functional outcomes in 9 patients with en bloc sacrectomy

\begin{tabular}{lcccc}
\hline \multicolumn{1}{c}{ Outcome } & 1 Mo & 3 Mos & 6 Mos & 12 Mos \\
\hline Nonambulatory & 5 & 1 & 0 & 0 \\
\hline Limited standing mobility & 2 & 0 & 0 & 0 \\
\hline Ambulate w/ device (walker) & 2 & 6 & 1 & 0 \\
\hline Ambulate independently & 0 & 0 & 4 & 5 \\
\hline Lost to FU & 0 & 2 & 4 & 4 \\
\hline
\end{tabular}

plications associated with rectal resection. These outcomes suggest that while local tissue rearrangement is a convenient strategy for soft-tissue coverage of the sacral wound, inherent deficiencies such as lack of tissue bulk or an area that allows for incomplete obliteration of dead space may incur higher risks of wound dehiscence and infection. On the other hand, the pedicled VRAM flap is associated with longer and more numerous surgeries, and with higher EBL. In light of these variables, we believe that a standardized protocol is needed to help surgeons determine patient candidacy for regional or distal flap reconstruction.

\section{Proposed Surgical Algorithm}

Our recommended algorithm for managing large sacral defects after total sacrectomy is summarized in Fig. 1. At our institution, the neurosurgical team often uses a singlestage, posterior approach for tumors limited to the lumbosacral spine, to minimize morbidity and number of operations; however, it has been noted that reconstruction of the resultant extensive soft-tissue defects is more challenging because the VRAM flap cannot be harvested. ${ }^{8}$ A 2-stage approach is used for patients with tumor invasion above the L5-S1 disc space, or with involvement of the rectum or ventral vasculature. ${ }^{6}$ For posterior-only operations, bilateral GM flaps are the initial choice for defect obliteration. In cases in which the GM was found to have insufficient area or bulk, PSM and LD flaps have been mobilized to supplement the gluteal tissue. Pelvic floor defects were subsequently reconstructed with bioprosthetic acellular dermal matrix rectal slings. For patients undergoing multistage operations, the pedicled VRAM flap has been the first choice in patients with no previous abdominal wall surgery. In fact, it is our opinion that the transpelvic VRAM flap is the gold standard for soft-tissue reconstruction after total sacrectomy, because it allows simultaneous achievement of 2 main goals: obliteration of dead space with vascularized tissue and prevention of posterior herniation of peritoneal contents. It should be noted that certain abdominal procedures requiring a simple midline incision may not preclude patients from receiving the VRAM flap, as long as the tissue and vascular supply remain intact. Preoperative CT angiography may be used to visualize vascularity to the flap muscle and myocutaneous perforating vessels to the overlying skin.

\section{Staged Approach}

Total en bloc sacrectomy is traditionally accomplished with a 2-stage approach, and can involve a multidisci- plinary team of oncological, neurological, vascular, orthopedic, and/or plastic and reconstructive surgeons. The first stage uses an anterior (intraperitoneal) approach to expose the ventral aspects of the tumor and to ligate the major vessels supplying it, which typically emanate from the internal iliac arteries. A pedicled VRAM flap based on the deep inferior epigastric vessels may be harvested at this time in anticipation of an extensive sacral defect. For the second stage, a posterior approach allows exposure and resection of the whole sacrum. The advantage of a 2-stage approach is greater access to structures anterior to the sacrum, which allows greater control of the neurovasculature and rectum. However, a single-stage, posterior-only approach reduces the number of operations, total operating time, and risk of wound complications secondary to iliac ligation. ${ }^{6}$ This strategy is contraindicated in the presence of 1) tumor invasion of the rectum requiring rectal diversion and resection; 2) caudal extension of the tumor beyond the L5-S1 disc space; or 3) involvement of the iliac vessels. Furthermore, a posterior-only approach precludes the use of the VRAM flap for subsequent defect repair.

\section{Perioperative Risk Factors}

Predisposing factors for wound complications include a history of DM, smoking, obesity (BMI $>30$ ), hypertension, hyperlipidemia, serum albumin $<3.5 \mathrm{~g} / \mathrm{dl}$, pre- or postoperative chemotherapy or RT, prior spinal or abdominal surgery, and presence of a colostomy. In our cohort, all patients had at least 1 documented risk factor prior to the operation. Five patients (56\%) had 1 risk factor, $1(11 \%)$ had 3 risk factors, $1(11 \%)$ had 4 risk factors, $1(11 \%)$ had 5 risk factors, and $1(11 \%)$ had 7 risk factors. Of the 4 patients who had more than 1 risk factor, 3 (75\%) developed early or delayed wound dehiscence. The fourth $(25 \%)$ patient's postoperative course was complicated by a partial small-bowel obstruction and delayed presacral abscess.

Six patients $(67 \%)$ were noted to have low $(<3.5 \mathrm{~g} / \mathrm{dl})$ perioperative serum albumin levels, which are a marker of nutritional status. ${ }^{33}$ Of these 6 patients, 4 (67\%) developed wound complications. Three patients underwent colostomy for bowel diversion prior to or at the time of the procedure. Only 1 of the 3 patients developed flap-related complications. However, bowel incontinence has been associated with prolonged hospital stays and delayed wound healing: ${ }^{6,20}$ our 3 patients with colostomy had a mean LOS of 38.7 days, compared with a mean LOS of 30 days for all patients.

\section{Soft-Tissue Reconstruction}

Complex wound closure after total sacrectomy must address the exposed posterior hardware in addition to any pelvic floor defects. Simple midline closures without proper obliteration of resultant dead space have been associated with complications such as hematoma, seroma, infection, fistula formation, and bowel obstruction; in contrast, recruitment of local or distal vascularized flaps can fill the dead space and prevent postoperative infection or dehiscence. ${ }^{15,28,29,33,35}$ Sacroperineal herniation is a rare but significant complication that results from increased intraabdominal pressure or denervation atrophy of the pelvic diaphragm. If the perineal muscles cannot be satisfactorily 


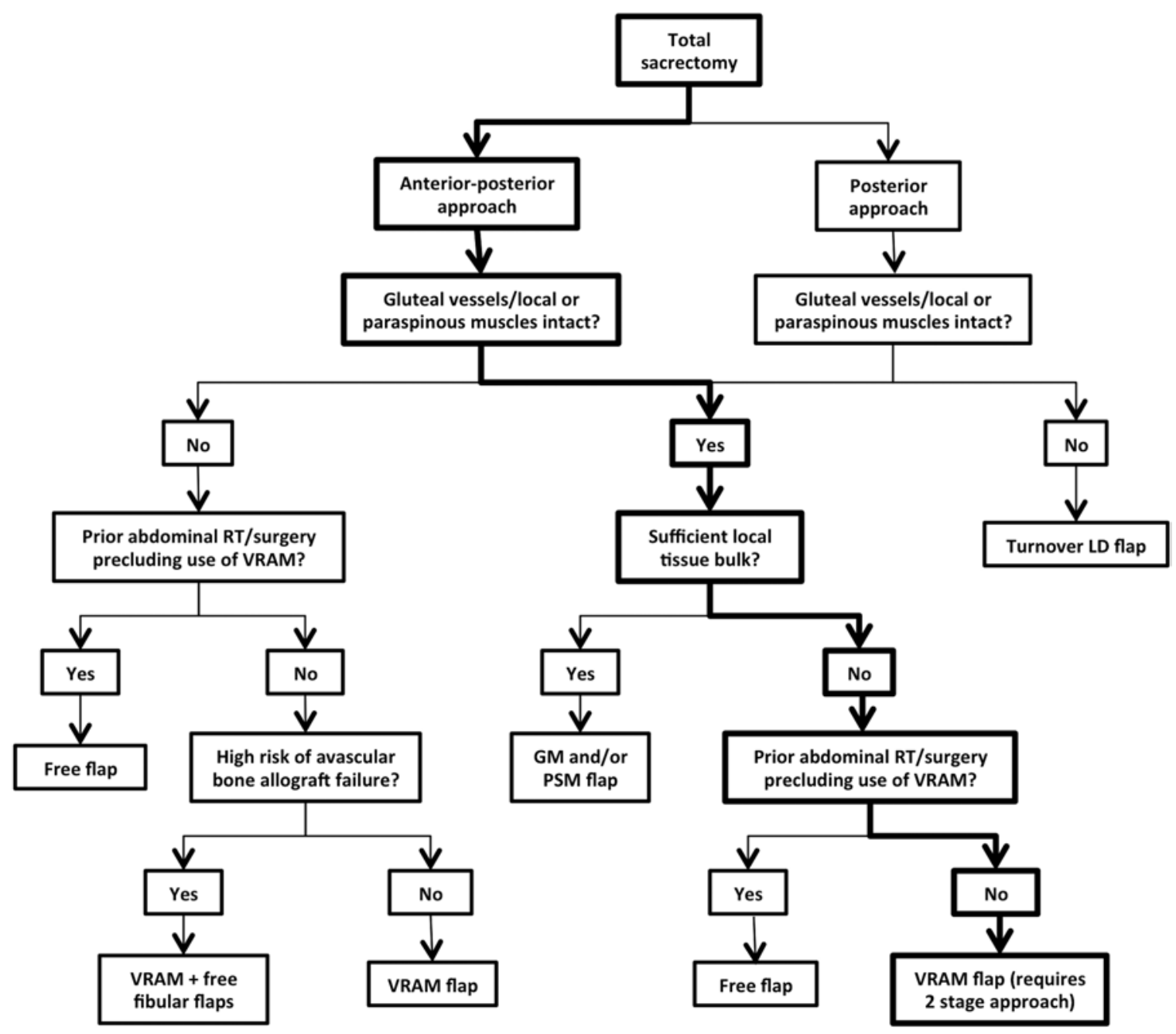

FIG. 1. Recommended algorithm for managing large sacral defects after total sacrectomy. Highlighted in bold is our gold standard approach performed using the pedicled VRAM flap.

approximated, a prosthetic rectal sling may be fashioned in conjunction with a local flap to create a tension-free barrier again posterior herniation of pelvic contents. Materials such as polypropylene mesh, ${ }^{1,14}$ porcine acellular dermal collagen, and human acellular dermal matrix have been described as viable options for these cases. Additionally, a transpelvic VRAM flap can recreate the pelvic diaphragm as it is passed posteriorly toward the sacral defect.

\section{Gluteal Flap}

In all patients, meticulous coverage of exposed implants and obliteration of dead space must be accomplished to maximize wound healing. Bilateral gluteal myocutaneous flaps are considered to be the first-line choice for lumbosacral defects, due to their proximity to the defect and robust blood supply from the hypogastric, superior gluteal, and inferior gluteal arteries. Flap designs vary depending on the amount of muscle available and viability of remaining vascular pedicles; ${ }^{24}$ multiple variations on the conventional rotation flap have been described in the literature, including the turnover or sliding flap and gluteal myocu- taneous V-Y advancement flaps. ${ }^{2,10,15,24}$ Reported rates of wound dehiscence and surgical-site infections range from $0 \%$ to $66.7 \%{ }^{8,23}$ Within our own cohort of 9 patients, we observed 4 cases $(44 \%)$ of postoperative wound infection and dehiscence.

Limitations that preclude the use of the gluteal flap include iatrogenic occlusion of the vessels or tumor invasion of the muscle or pedicle. Native blood supply to the gluteal arteries is more likely to be preserved with a posterioronly approach, as this avoids the routine sacrifice of the internal iliac vessels. Additionally, en bloc sacrectomy may include resection of involved gluteal muscle, diminishing the volume of tissue left to fill the resultant dead space. For cases in which the GM flap is found to have insufficient bulk-especially in elderly patients with attenuated tissue-regional flaps of the posterior thigh, PSM, or pedicled LD may be mobilized to obliterate the dead space. ${ }^{26,33}$ A "reverse turnover" LD muscle flap covered with splitthickness skin grafts has been successfully used as a principal flap for lumbosacral defects.9,22,37 The LD muscle is detached at its insertion at the humerus, separating it from the thoracodorsal artery, vein, and nerve. The belly of the 
muscle is then reversed and inset into the wound, with vascular supply based on the secondary segmental parasinous vessels. ${ }^{33}$ It is our recommendation that the reverse turnover LD flap be considered when gluteal vessels are not intact or local muscles are insufficient for coverage of the defect (Fig. 1). Of the 7 patients in our study who underwent gluteal muscle flap reconstruction, 4 required additional bilateral PSM flaps, and 1 required bilateral LD flaps.

\section{Omental Flap}

The omental flap is another reliable option for supplemental bulk in cases of local tissue deficit. In a 2003 report by Diaz et al., 4 of 6 patients receiving bilateral gluteal advancement flaps required an additional omental flap to obliterate the remaining dead space. ${ }^{11}$ The primary criteria for use of the omental flap for coverage of sacral instrumentation include irradiated, divided, or otherwise nonviable local musculature. The greater omentum provides a wellvascularized, pedicled flap that can be used in conjunction with or as a replacement for an inadequate local flap. With an area averaging $400 \mathrm{~cm}^{2}$ and a mean pedicle caliber of $2 \mathrm{~mm}$, the omental flap can be rotated across the ventral surface of the body and passed circumferentially toward the sacral defect. ${ }^{11}$ The omentum is preferred for smaller sacral defects (i.e., smaller than the surgeon's fist), whereas a rectus abdominis flap may be required for larger reconstructions. ${ }^{27}$ Benefits of using the omental flap include the tissue's absorptive and angiogenic qualities as well as its capacity as a substrate for adhesion formation and defense against infection or irradiation, all of which can accelerate wound healing. ${ }^{25,28}$ The main disadvantage is the need for an anterior approach for flap harvest, which may require an additional stage or intraoperative positioning change. ${ }^{33}$ The use of the omental flap is contraindicated in the case of prior radiation or resection of abdominal tissue, or morbid obesity. Previously described complications include wound dehiscence, seroma, deep wound infection, chylous leak, ileus, ventral herniation, and presacral abscess. ${ }^{11,28,33}$

\section{Free Flaps}

Free soft-tissue flaps are generally less favored due to the quality of available recipient vessels, which are small in caliber, short, or anatomically difficult to access. In cases in which the superior and inferior gluteal arteries have been compromised by prior irradiation, surgery, or embolization, the femoral and thoracodorsal vessels may be used as recipients, but require long vein grafts. For extensive sacral defects, the use of a free LD flap may be preferred over a pedicled flap, because the pedicle limits mobility and reach. ${ }^{33}$ Gaster et al. advocated the use of a free transfer rectus abdominis myocutaneous flap as an option with sufficient bulk and area to cover large sacral wounds without the need for additional regional flaps. ${ }^{17}$ However, free flaps are a last resort for sacrectomy defect coverage, and should not be used if local or regional flaps are available.

\section{The VRAM Flap}

The transpelvic VRAM flap is the preferred approach at our institution. Although it requires a 2-stage approach, it not only provides sufficient tissue bulk to obliterate dead space, but also offers the added benefit of recreating the pelvic floor because it is passed retroperitoneally, thereby obviating the need for a bioprosthetic sling. A portion of the flap can be placed posteriorly to the rectum, preventing herniation.

The pedicled VRAM flap contains variable amounts of skin, adipose tissue, and the rectus muscle based on the deep epigastric artery and vein. The flap is harvested during the first stage of the procedure, after anterior laparotomy and ligation of the internal iliac arteries. Flap area is maximized in anticipation of a large defect, and placed in the pelvis until the second stage. After posterior sacral amputation, the flap is retrieved from the pelvis through the posterior wound and inserted over the defect (Fig. 2). The flap can be used to reconstruct soft-tissue volumetric loss and cutaneous skin from the sacral region. The advantages of this flap are that it does not require microvascular techniques and can supply a large surface area and sufficient bulk for wound coverage and dead space obliteration. This is especially important considering the large volume of tissue that may be removed during tumor resection (Fig. 3 ). Furthermore, the pedicled VRAM acts as an obturator between the peritoneal cavity and the posterior wound, preventing bowel from herniating into the posterior wound adjacent to the thecal sac and spinal hardware.

The VRAM flap is generally used in cases in which local flaps are contraindicated. In a study of 27 flap reconstructions after total sacrectomy, Miles et al. proposed that the VRAM flap be considered in patients with prior sacral irradiation or surgery, but avoided in patients who have undergone laparotomy or ostomy. ${ }^{26}$ In contrast, Glatt et al. reported the successful use of the VRAM flap in 12 patients, 5 of whom had prior or concurrent laparotomy with ostomy, and 2 of whom had prior abdominal RT and surgery. The authors suggested that the transabdominal VRAM may be the most reliable and straightforward

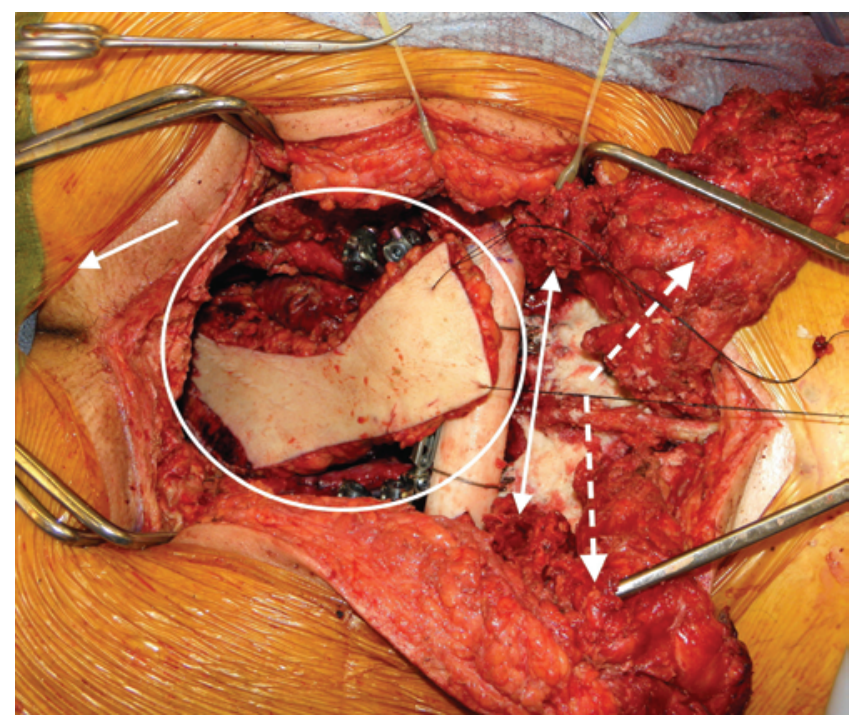

FIG. 2. Intraoperative photograph showing pedicled VRAM flap pulled through abdominal cavity during second stage of sacral resection. Dotted arrow indicates mobilized paraspinous tissues, double-headed arrow indicates the transiliac bar, circle indicates the VRAM flap, and singleheaded arrow indicates the anus. Figure is available in color online only. 


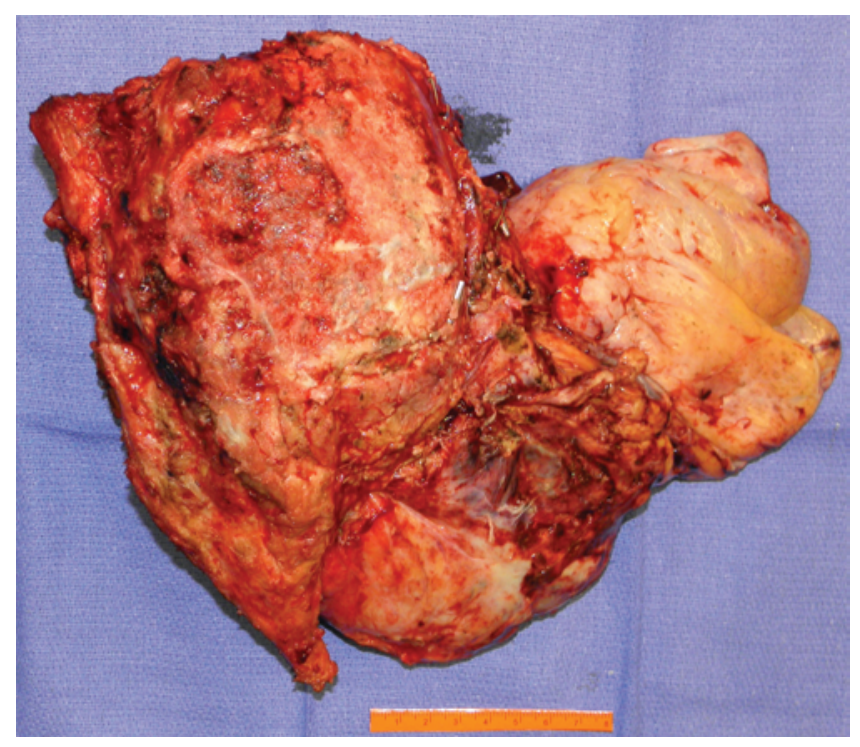

FIG. 3. Photograph showing en bloc resected tumor specimen of locally invasive rectal carcinoma involving entire sacrum, rectum, bladder, prostate, and left iliac vein. Tape measure at bottom marks $8 \mathrm{~cm}$. Figure is available in color online only.

choice for flap reconstruction of large sacral and perineal defects following total sacrectomy. Previously described complications of VRAM reconstruction of sacral defect include wound dehiscence, seroma, flap necrosis, and enterocutaneous fistula. ${ }^{16,18}$ In our series, postoperative complications following VRAM reconstruction included hemodynamic instability after massive intraoperative blood loss and small-bowel obstruction after rectal resection of primary tumor. Hemodynamic instability was most likely related to prolonged tumor resection secondary to greater tumor burden and invasion, which warranted the multistage sacrectomy with VRAM flap approach. Because neither patient experienced local flap failure or local wound complications, the distal complications may be attributed to the use of the AP approach and multiple stages, as opposed to the use of the flap itself.

\section{Combined VRAM and Free Fibular Flaps}

The combined use of both pedicled VRAM flap and free fibula has been described as an alternative to avascular bone grafting in patients with a high risk of delayed union (Fig. 1) ${ }^{16}$ Incorporation of avascular allograft bone takes several years, and has been associated with significant rates of delayed or failed union, pseudarthrosis, and graft resorption., ${ }^{7,30}$ In our study, we observed 1 case of femoral bone graft failure at 1 month postoperatively. The patient was taken back to the operating room for graft removal, sacral wound washout, and VAC placement. In contrast, vascularized bone flaps have been shown to have superior mechanical and functional outcomes, including greater stiffness and stability, and higher rates of arthrodesis, with union times ranging from 2 to 10 months., 516,27

The combined VRAM and free fibular technique is complicated and has a high morbidity rate. Garvey et al. noted that patients often required a third stage for fibular flap harvest, because they were too hemodynamically labile by the end of the second stage. However, ligating the internal iliac arteries during the second stage can significantly lower blood loss and prevent the need for an additional operation. In addition, union times have been excellent, and the use of vascularized free bone flaps may be warranted in cases of failed arthrodesis secondary to infection or neurofibromatosis, failed fusion secondary to neuromuscular disease, or poor osseous and soft-tissue beds secondary to RT. ${ }^{16}$

\section{Total En Bloc Sacrectomy With Pedicled VRAM Flap Reconstruction: a Worthwhile Endeavor?}

Large sacral tumors pose a unique oncological challenge. Surgical management requires complex and often multiple procedures with a well-coordinated, interdisciplinary team. Resection may be a lengthy procedure, with significant blood loss and peri- or postoperative morbidity. Surgeons must be mindful of the risks associated with this procedure, which include permanent damage to motor or sensory nerves, or may result in bowel, bladder, or sexual dysfunction. ${ }^{19,31,38}$ Therefore, surgical candidates must be carefully selected after taking into consideration any comorbidities, anatomical characteristics, and tumor biology. ${ }^{31}$ Total en bloc sacrectomy, which is recommended for tumors that involve $\mathrm{S}-1$ or the sacroiliac joints, is the most drastic form of resection for sacral tumors, and is also associated with greater morbidity and cost. ${ }^{3,11}$ In a comparative study of proximal (above S-2) versus distal sacral resections, Diaz et al. found that average hospital costs were $\$ 28,800$ for proximal resections, compared with $\$ 7500$ for distal resections. Additionally, major complications were seen in $85 \%$ versus $29 \%$ of patients with proximal versus distal sacrectomies, respectively. ${ }^{11}$ The authors therefore suggested that proximal osteotomies be considered for patients with a realistic cure potential.

Despite the high monetary costs, we believe that total en bloc sacrectomy is a worthwhile endeavor in select cases in which surgical cure with R0 resection (complete resection with no residual tumor cells as confirmed by microscopic examination) or long-term survival and functionality is possible. Sacrectomy is contraindicated in aggressive metastatic disease (such as lung cancer) involving multiple vertebrae or internal organs, or in patients with significant comorbidities or poor functionality. ${ }^{14}$ However, patients with less aggressive disease such as metastatic breast and prostate cancer may benefit from surgical reduction of overall tumor burden. ${ }^{32}$ Long-term survival may be feasible in select patients with recurrent metastatic rectal cancer if margin-negative resection is achieved. ${ }^{12,36}$ Furthermore, giant cell tumors, ${ }^{19}$ primary sacral osteosarcomas, ${ }^{34}$ and sacral chordomas ${ }^{14}$ have been shown to be amenable to sacral resection, with reports of prolonged survival and functional recovery.

In addition to careful patient selection, operative strategies may be modified to minimize patient risk and hospital costs. ${ }^{3,29}$ A single-center, retrospective study of resource management and patient outcomes by Brown et al. found that the use of staged procedures (anterior followed by posterior approach) resulted in decreased postoperative transfusions $(\mathrm{p}=0.03)$, intensive care unit stays $(\mathrm{p}=0.03)$, time on a ventilator $(\mathrm{p}<0.01)$, and morbidity $(\mathrm{p}<0.01)$, 
compared with a nonstaged, posterior-only approach. Likewise, the mean total inpatient costs were lower by an average of $\$ 89,132$ for an AP approach (the mean costs of nonstaged vs staged procedures were \$179,899 and \$4661, respectively). ${ }^{3}$ Postoperative complications such as wound infection and dehiscence can add considerable costs, especially if a reoperation for debridement and washout with or without VAC is required. Therefore, it is to both the patient's and the hospital's benefit to use a soft-tissue reconstruction strategy that will adequately obliterate the sacral defect and prevent wound complications as well as mechanical complications such as bowel herniation. Bowel obstruction secondary to herniation into the postsacral space is a potentially life-threatening condition, and the associated costs of medical and surgical management can be significant. For this reason, it is our opinion that the VRAM is the best option for reconstruction after a staged total sacrectomy.

In our patient series, neither of the 2 patients who received a VRAM flap had wound complications, whereas 4 cases with local flaps (bilateral GM with or without PSM flaps) were complicated by wound dehiscence, 3 of whom required surgical debridement. Wound infection also occurred in 2 patients in whom reconstruction with local tissue rearrangement was performed. Although the use of a VRAM flap was associated with postoperative hemodynamic instability, this was most likely related to a higher initial tumor burden, leading to prolonged oncological resection during which the majority of blood loss occurred. Further studies of patient outcomes, resource utilization, and hospital expenses will be needed to provide a more comprehensive understanding of the risks and benefits of this massive undertaking.

\section{Conclusions}

Our experience suggests that the GM and pedicled VRAM flaps are reliable options for reconstruction of the pelvic diaphragm and obliteration of dead space overlying the spinal hardware. In patients undergoing a single-stage, posterior-only operation, GM flaps are generally used. This method requires the presence of intact gluteal vessels, sufficient local tissue bulk, and the use of a prosthetic rectal sling to prevent sacral herniation. For multistage AP operations, the pedicled VRAM flap is the gold standard because it reliably provides sufficient tissue volume while also creating a barrier between the pelvic viscera and the sacral defect, thereby obviating the need for a mesh sling. Although complications are expected within the first 6 weeks of the postoperative period, patients may also expect to be mobile and ambulatory within 1 year of the procedure, provided that they do not experience a tumor recurrence or hardware failure.

\section{Acknowledgment}

Data collection for this work was supported by a grant from AOSpine International.

\section{References}

1. Atkin G, Mathur P, Harrison R: Mesh repair of sacral hernia following sacrectomy. J R Soc Med 96:28-30, 2003
2. Ay $\mathrm{A}$, Aytekin $\mathrm{O}$, Aytekin A: Interdigitating fasciocutaneous gluteal V-Y advancement flaps for reconstruction of sacral defects. Ann Plast Surg 50:636-638, 2003

3. Brown MJ, Kor DJ, Curry TB, Warner MA, Rodrigues ES, Rose SH, et al: Sacral tumor resection: the effect of surgical staging on patient outcomes, resource management, and hospital cost. Spine (Phila Pa 1976) 36:1570-1578, 2011

4. Chang CY, Li MC, Liao SL, Huang YL, Shen CC, Pan HC: Prognostic and clinical implication of IL-6 expression in glioblastoma multiforme. J Clin Neurosci 12:930-933, 2005

5. Chang DW, Friel MT, Youssef AA: Reconstructive strategies in soft tissue reconstruction after resection of spinal neoplasms. Spine (Phila Pa 1976) 32:1101-1106, 2007

6. Clarke MJ, Dasenbrock H, Bydon A, Sciubba DM, McGirt MJ, Hsieh PC, et al: Posterior-only approach for en bloc sacrectomy: clinical outcomes in 36 consecutive patients. Neurosurgery 71:357-364, 2012

7. Coric D, Branch CL Jr, Jenkins JD: Revision of anterior cervical pseudoarthrosis with anterior allograft fusion and plating. J Neurosurg 86:969-974, 1997

8. Dasenbrock HH, Clarke MJ, Bydon A, Witham TF, Sciubba DM, Simmons OP, et al: Reconstruction of extensive defects from posterior en bloc resection of sacral tumors with human acellular dermal matrix and gluteus maximus myocutaneous flaps. Neurosurgery 69:1240-1247, 2011

9. de Fontaine S, Gaede F, Berthe JV: The reverse turnover latissimus dorsi flap for closure of midline lumbar defects. J Plast Reconstr Aesthet Surg 61:917-924, 2008

10. Di Mauro D, D'Hoore A, Penninckx F, De Wever I, Vergote I, Hierner R: V-Y Bilateral gluteus maximus myocutaneous advancement flap in the reconstruction of large perineal defects after resection of pelvic malignancies. Colorectal Dis 11:508-512, 2009

11. Diaz J, McDonald WS, Armstrong M, Eismont F, Hellinger M, Thaller S: Reconstruction after extirpation of sacral malignancies. Ann Plast Surg 51:126-129, 2003

12. Dozois EJ, Privitera A, Holubar SD, Aldrete JF, Sim FH, Rose PS, et al: High sacrectomy for locally recurrent rectal cancer: can long-term survival be achieved? J Surg Oncol 103:105-109, 2011

13. Ehrler DM, Vaccaro AR: The use of allograft bone in lumbar spine surgery. Clin Orthop Relat Res (371):38-45, 2000

14. Fourney DR, Gokaslan ZL: Current management of sacral chordoma. Neurosurg Focus 15(2):E9, 2003

15. Furukawa H, Yamamoto Y, Igawa HH, Sugihara T: Gluteus maximus adipomuscular turnover or sliding flap in the surgical treatment of extensive sacral chordomas. Plast Reconstr Surg 105:1013-1016, 2000

16. Garvey PB, Clemens MW, Rhines LD, Sacks JM: Vertical rectus abdominis musculocutaneous flow-through flap to a free fibula flap for total sacrectomy reconstruction. Microsurgery 33:32-38, 2013

17. Gaster RS, Bhatt KA, Shelton AA, Lee GK: Free transverse rectus abdominis myocutaneous flap reconstruction of a massive lumbosacral defect using superior gluteal artery perforator vessels. Microsurgery 32:388-392, 2012

18. Glatt BS, Disa JJ, Mehrara BJ, Pusic AL, Boland P, Cordeiro PG: Reconstruction of extensive partial or total sacrectomy defects with a transabdominal vertical rectus abdominis myocutaneous flap. Ann Plast Surg 56:526-531, 2006

19. Gokaslan ZL, Romsdahl MM, Kroll SS, Walsh GL, Gillis TA, Wildrick DM, et al: Total sacrectomy and Galveston Lrod reconstruction for malignant neoplasms. Technical note. J Neurosurg 87:781-787, 1997

20. Guo Y, Palmer JL, Shen L, Kaur G, Willey J, Zhang T, et al: Bowel and bladder continence, wound healing, and functional outcomes in patients who underwent sacrectomy. J Neurosurg Spine 3:106-110, 2005

21. Junge K, Krones CJ, Rosch R, Fackeldey V, Schumpelick V: 
Mesh reconstruction preventing sacral herniation. Hernia 7:224-226, 2003

22. Kalaaji A, Rosenberg BE, Olstad OA, Høiness P, Røise O: "Reversed turnover" latissimus dorsi muscle flap for reconstruction of a deep sacral defect after a severe pelvic fracture. Scand J Plast Reconstr Surg Hand Surg 40:186-188, 2006

23. Kimura H, Nasu W, Kashiwa K, Kobayashi S: Reconstruction of the sacral region using the lumbo-gluteal sensory flap. J Plast Reconstr Aesthet Surg 66:239-242, 2013

24. Koh PK, Tan BK, Hong SW, Tan MH, Tay AG, Song C, et al: The gluteus maximus muscle flap for reconstruction of sacral chordoma defects. Ann Plast Surg 53:44-49, 2004

25. Micheau P: [The greater omentum. Its role in reconstructive plastic surgery.] Ann Chir Plast Esthet 40:192-207, 1995 (Fr)

26. Miles WK, Chang DW, Kroll SS, Miller MJ, Langstein HN, Reece GP, et al: Reconstruction of large sacral defects following total sacrectomy. Plast Reconstr Surg 105:23872394, 2000

27. Moran SL, Bakri K, Mardini S, Shin AY, Bishop AT: The use of vascularized fibular grafts for the reconstruction of spinal and sacral defects. Microsurgery 29:393-400, 2009

28. O'Shaughnessy BA, Dumanian GA, Liu JC, Ganju A, Ondra SL: Pedicled omental flaps as an adjunct in the closure of complex spinal wounds. Spine (Phila Pa 1976) 32:30743080, 2007

29. Ramamurthy R, Bose JC, Muthusamy V, Natarajan M, Kunjithapatham D: Staged sacrectomy-an adaptive approach. J Neurosurg Spine 11:285-294, 2009

30. Samartzis D, Shen FH, Matthews DK, Yoon ST, Goldberg EJ, An HS: Comparison of allograft to autograft in multilevel anterior cervical discectomy and fusion with rigid plate fixation. Spine J 3:451-459, 2003

31. Sciubba DM, Nelson C, Gok B, McGirt MJ, McLoughlin GS, Noggle JC, et al: Evaluation of factors associated with postoperative infection following sacral tumor resection. J Neurosurg Spine 9:593-599, 2008

32. Shehadi JA, Sciubba DM, Suk I, Suki D, Maldaun MV, McCutcheon IE, et al: Surgical treatment strategies and outcome in patients with breast cancer metastatic to the spine: a review of 87 patients. Eur Spine J 16:1179-1192, 2007
33. Singh K, Samartzis D, Heller JG, An HS, Vaccaro AR: The management of complex soft-tissue defects after spinal instrumentation. J Bone Joint Surg Br 88:8-15, 2006

34. Spiegel DA, Richardson WJ, Scully SP, Harrelson JM: Longterm survival following total sacrectomy with reconstruction for the treatment of primary osteosarcoma of the sacrum. A case report. J Bone Joint Surg Am 81:848-855, 1999

35. Stener B, Gunterberg B: High amputation of the sacrum for extirpation of tumors. Principles and technique. Spine (Phila Pa 1976) 3:351-366, 1978 (Erratum in Rev Chir Orthop 73:following 217, 1987)

36. Yamada K, Ishizawa T, Niwa K, Chuman Y, Aikou T: Pelvic exenteration and sacral resection for locally advanced primary and recurrent rectal cancer. Dis Colon Rectum 45:1078-1084, 2002

37. Zakaria Y, Hasan EA: Reversed turnover latissimus dorsi muscle flap for closure of large myelomeningocele defects. J Plast Reconstr Aesthet Surg 63:1513-1518, 2010

38. Zileli M, Hoscoskun C, Brastianos P, Sabah D: Surgical treatment of primary sacral tumors: complications associated with sacrectomy. Neurosurg Focus 15(5):E9, 2003

\section{Author Contributions}

Conception and design: Sacks, Kim, Coon, Redett. Acquisition of data: Sacks, Kim, Pang, Christensen, Coon, Zadnik, Wolinsky, Gokaslan, Bydon, Sciubba, Witham, Redett. Analysis and interpretation of data: Kim. Drafting the article: Kim. Critically revising the article: Sacks, Kim, Pang, Christensen, Zadnik, Wolinsky, Gokaslan, Redett. Reviewed submitted version of manuscript: Sacks, Pang, Christensen, Coon, Zadnik, Wolinsky, Gokaslan, Bydon, Sciubba, Witham, Redett. Approved the final version of the manuscript on behalf of all authors: Sacks. Study supervision: Sacks.

\section{Correspondence}

Justin M. Sacks, Department of Plastic and Reconstructive Surgery, Johns Hopkins Outpatient Center, 601 N. Caroline St., Ste. 2114C, Baltimore, MD 21287. email: jmsacks@jhmi.edu. 